\title{
The Impact of Accessibility of Urban Central Parks on Housing Prices of Fuzhou
}

\author{
Wenhao Tan ${ }^{1}$, Linfeng $\operatorname{Liu}^{1}$, Yage $\mathrm{Cui}^{1}$, Jintao $\mathrm{Chen}^{1}$, Feng Lin ${ }^{1}$, Yingping Zhong ${ }^{1}$, Hui Pan*12 \\ ${ }^{1}$ Fujian Agriculture and Forestry University, Fuzhou, China \\ ${ }^{2}$ Minjiang University, Fuzhou, China
}

\begin{abstract}
The paper analysed the impact of accessibility of urban parks within the third ring on housing prices of Fuzhou through network analysis and SPSS correlation analysis based on data of sources like remote-sensing image, web crawling and urban road network, discovering that: (1)The density of residence within the third ring of Fuzhou decreases from the centre to the edges; (2) The housing price, ranging between 18000 and $28000 \mathrm{RMB} / \mathrm{m}^{2}$, peaks in the city centre; (3) For houses within the third ring of Fuzhou, Gulou District enjoys the greatest access to urban parks while Cangshan District is the poorest in this regard. (4) The residence within the third ring of Fuzhou could be rated as A or A- in terms of access to urban parks, with an overall excellence performance; (5) The walking distance to the parks is significantly correlated with the housing price. The shorter the distance, the higher the price. Regarding this, the paper proposed the following suggestions: (1) Revise the routes to Gaogai Mountain Park by increasing entrances and exists to improve its accessibility; (2) Improve the transportation network and increase footpaths to the park, thus shortening the distance between the park and the surrounding residences.
\end{abstract}

\section{Introduction}

Since the reform and opening-up, China has been witnessing a rapid rate of urbanization, which has reached $59.58 \%$ by the end of 2018 , a level well above the average level in the world ${ }^{[1]}$. Urban public facilities are challenged by increasing urban population, people's growing needs to improve living qualities and surroundings. As a major public facility, the wellmanaged urban central parks usually covers a wide area of grassland with abundant resources, providing a perfect place for outdoor activities ${ }^{[2]}$. Accessibility usually refers to the level of efforts that people need to make to get to a specific destination, which is measured by distance, time consumption and expenses ${ }^{[3]}$. The accessibility of central parks has a big impact on the life of urban dwellers. By studing literatures home and abroad, scholars are focusing on the social effects of the accessibility of urban parks ${ }^{[4]}$, the relationship between grassland and population, social fairness and services ${ }^{[5]}$, comparision of the accessibility of different types of gransslands ${ }^{[6,7]}$, and how to measure the accessibility to grasslands in real scenarios ${ }^{[8,9]}$. Coombes et al. explored the connection between residents' physical health and the accessibility of green space ${ }^{[10]}$; Dai analyzed differences among groups in terms of accessibility of green space ${ }^{[11]}$; Wendel et al. learned about urban residents' preferences on green space and their difficulties in transportation through sample survey and semi-structured interviews to find out the accessibility and utilization of urban green space in the midst of rapid urbanization ${ }^{[12]}$. Coutts et al. studied the impact of accessibility of urban green space on health ${ }^{[13]}$.The major approaches to measure the accessibility of green space include: buffer analysis, minimum distance calculation, cost-distance calculation, gravity model ${ }^{[14,15]}$ and Gaussian two-step floating catchment area ${ }^{[16]}$. There have been few studies on the impact of accessibility of urban central parks on housing prices through network analysis.

Researchers believed that the road-based network analysis could better reflect the process of how residents access the park, providing more accurate and objective analysis on the accessibility of parks ${ }^{[17]}$. Therefore, the paper adopted network analysis based on data from web crawling and Fuzhou urban parks to study the accessibility of urban parks on housing prices. By transforming the study into quantitative data, it provides references for future planning on urban residence, which is important to people's well-being.

\section{Survey Region}

The area within the third ring of Fuzhou covers the Cangshan Distrcit, Gulou District, Ji'an District, Taijiang District, making it the most populous area with abundant public facilities such as parks and transportation network ${ }^{[18-19]}$. For this reason, it is selected as the region for survey.

\footnotetext{
* Corresponding author's e-mail: 332088289@qq.com
} 


\section{Research Method and Procedures}

\subsection{Source of data}

The data is obtained from sources including Anjuke POI, park survey and urban transportation network: (1) Information on the housing price, name of the community as well as the longitude and latitude of the houses for sale within the third ring of Fuzhou were crawled on the website of Anjuke, followed by correction of the data. First, exclude error data by statistics analysis; Next, data on the region from 58.com, ganji.com and fang.com was crawled for analysis while the missing data was supplemented. Finally, the data was imported into ArcGis10.5 for spatial correction by highresolution remote sensing image and Baidu online map, where the wrong data was deleted and the missing data was completed. (2) Data on a total of 55 parks was obtained from the Website of Fuzhou Garden, through which 22 parks covering more than $10 \mathrm{hm} 2$ were selected based on the Urban Green Space Classification Standard (CJJ/T 85-2017). The visitors flow rate was accurately recorded by field handled GPS. (3) The high-resolution remote sensing images of 2019 were obtained and imported into ArcGIS10.5 for artificial interpretation based on the overall urban plan of Fuzhou between 2011 and 2020, through which the road information was obtained. Meanwhile, the location of the parks was imported for data supplement.

\subsection{Construction of network analysis model based on database}

The park and road network database are established based on the requirements of ArcGIS network analyst module, consisting of the centres, links, nodes and impedance that reflect the influence of urban green space (centre) along the traffic network under a certain resistance value (time, distance, cost, etc.). The "New Network Data Set" of the ArcGIS was used to construct the spatial resistance model for network analysis, followed by the establishment of the service analysis layer, where the shortest route from the facility point to the event point along the road network was generated by setting the resistance value of road time (road length/passage speed) ${ }^{[20]}$. The park database includes the name, coverage, entrance and other elements of the park, with the entry point of the park serving as the facility point of the network. The database of road network includes the route, name, classification, traffic speed and traffic time of the road. The road network line is established with the central line of the road as the base, the intersection of the breakline as the junction node to simulate the time consumption of traffic at the crossroads of the road network. For residents mainly travelling on foot, their walking speed is set at $5 \mathrm{~km} / \mathrm{h}$ ${ }^{\text {[21] }}$. A waiting time of $0.5 \mathrm{~min}$ is set at the road node ${ }^{\text {[22] }}$

\subsection{Calculation of the accessibility of parks}

Based on the nearest facility point tool in ArcGIS 10.5 Network Analysis, the park is set as the facility point and residential houses are taken as the event point to calculate the shortest path between the park and residence, which is considered the quantitative index measuring the shortest path to the park.

\subsection{Correlation analysis between the accessibility of parks and housing prices}

SPSS software was used to analyse the correlation between the shortest path to parks and the housing price in an effort to study the influence of the accessibility of parks on housing price.

\section{Result Analysis}

\subsection{Location-based housing price variation}

Based on Figure 1, it is known that the urban residence of Fuzhou is distributed in a circular pattern with the city centre as the core. The density of houses decreases from the first ring to the third ring, which peaks at the east side of Xihu Park in Gulou District, probably because the area has a smaller coverage and greater population compared with other administrative districts. The Gulou District is followed by Taijiang District, Ji'an District and Cangshan District in terms of population density, while Cangshan District harbors the most land resources within the third ring of Fuzhou. However, schools such as Fujian Agriculture and Forestry University, Fujian Engineering College and Fujian Normal University are located in the district, all occupying a vast area of land. In addition, lands like Feifeng Mountain and Gaogai Mountain are difficult to be exploited. All these factors contribute to the less population and smaller scale of residence distribution of the district.

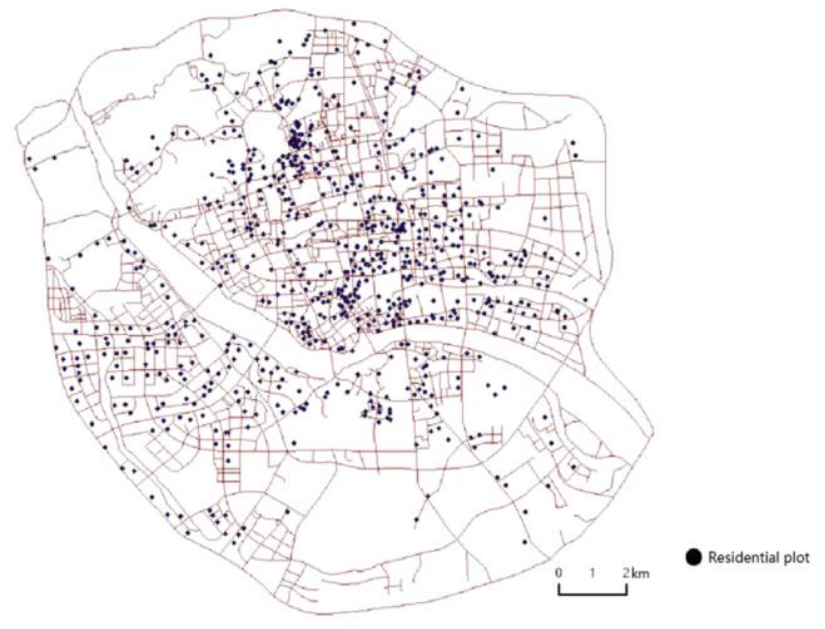

Figure 1 Distribution of Residence within the Third Ring of Fuzhou

The housing prices within the third ring of Fuzhou averages at RMB $25873.60 / \mathrm{m}^{2}$ between RMB 
$10009.38 / \mathrm{m}^{2}$ and RMB $89227.64 / \mathrm{m}^{2}$. Analysis of the housing prices through ArcGIS based on the inverse distance weighting method revealed that the majority of the residential houses are priced between $18000 / \mathrm{m}^{2}$ and $28000 / \mathrm{m}^{2}$ while a smaller part of houses is priced between $28000 / \mathrm{m}^{2}$ and $36000 / \mathrm{m}^{2}$, constituting more than half of the survey subjects. The housing prices peaks at the city centre and decreases along the outside rings. Gulou District, as the city centre, is the most economically developed district of Fuzhou, with the highest population and best facilities. For communities, the Jintai Community of Gulou District has the highest housing price at $89227.64 / \mathrm{m}^{2}$.

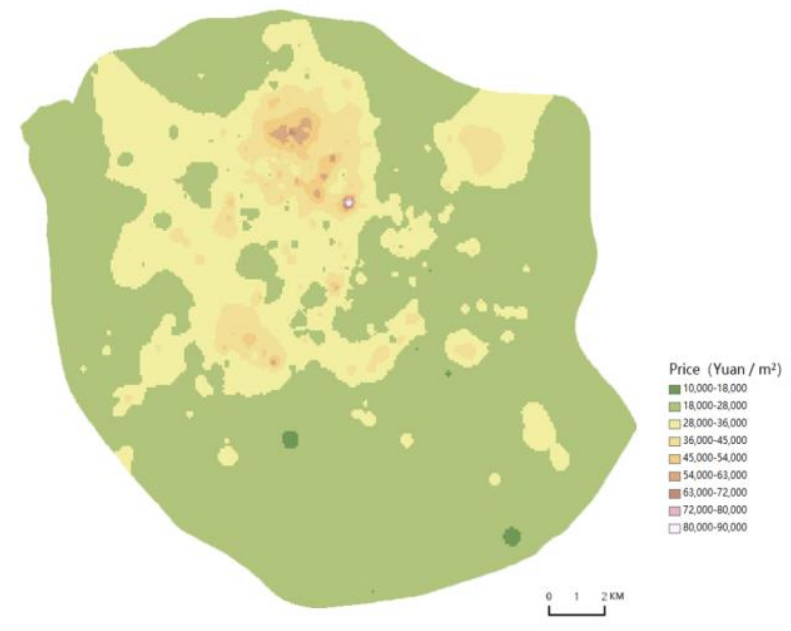

Figure 2 Location-based Housing Prices within the Third Ring of Fuzhou

\subsection{Analysis of green space accessibility}

Based on the Network Analysis module of ArcGIS, all the entry and exit points of the parks are taken as the facility point while all the residential houses are taken as the event point to calculate the shortest path between each community and the park, generating a total of 716 shortest paths. The time consumed in each shortest path is graded based on five classes: 1. Excellent (0.00528$0.312096) ; 2$. Good (0.312097-0.618912); 3. So-so (0.618912-0.925728); 4. Poor (0.925728-1.232545); 5. Very poor (1.232545-1.539361), as shown in Figure 3. From the figure, residence with good accessibility to the parks are many scattered in the northeast side of Xihu Park and the south side of Wenquan park in Gulou District due to the dense road network of Gulou District and residential houses as well as the rational planning of entry and exit points of the two parks. For Taijiang District, residential houses with good accessibility to the parks concentrate in the area near Minjiang North Park and Guanggang Park, both of which are belt-shaped parks with more entry and exit points and a wider range of coverage, making it more accessible for the residents. Residential houses of Cangshan District are mainly graded as Good and So-so, of which the ones with poor accessibility to parks mainly concentrate on Fuwan Road of Cangshan District as a result of poor infrastructure and scattered road network. In addition, Gaogai
Mountain Park has only one entry point on the northeast side, making it difficult to access.

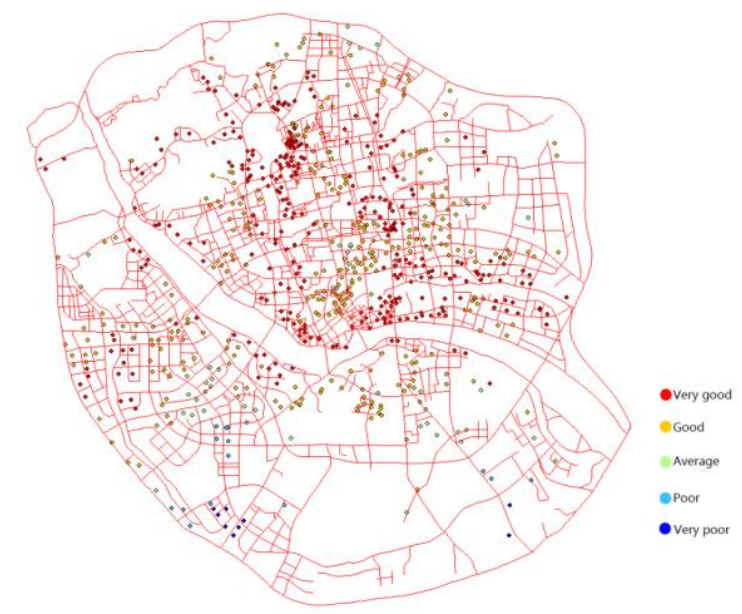

Figure 3 Acessibility Grade of Parks for Residential Houses within the Third Ring of Fuzhou

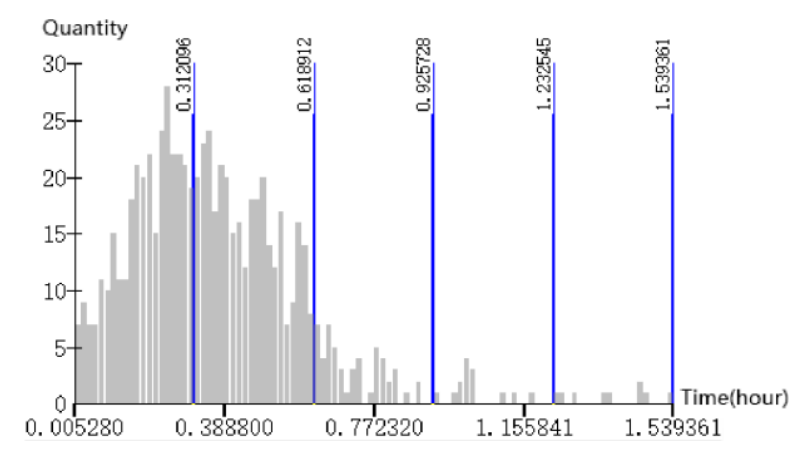

Figure 4 Quantitative Distribution of Park Accessibility for Residential Houses within the Third Ring of Fuzhou

From Figure 4, it is known that the residential houses within the third ring of Fuzhou are mainly graded as Very good and Good, with an average time consumption on the shortest path of $0.374 \mathrm{~h}$, which is considered acceptable.

\subsection{The impact of green space accessibility on housing prices}

The residential housing price and the corresponding walking time via the shortest path are imported into the SPSS for bivariate correlation analysis, as shown in Table 1, which reveals a Pearson correlation between the walking time and the housing price of -0.205 , i.e. the shorter the walking time, the higher the house prices, which were significantly related.

Table 1 Correlation Analysis of Green Space Accessibility and Housing Prices

\begin{tabular}{|cc|c|c|}
\hline & & Walk time & House price \\
\hline Walk time & Pearson Correlation & 1 & $-.205^{* *}$ \\
& Sig. (2-tailed) & & .000 \\
$\mathrm{~N}$ & 716 & 716 \\
\hline
\end{tabular}




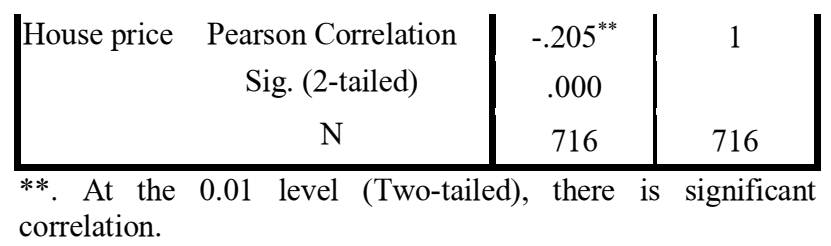

\section{Conclusion and Suggestion}

The paper studied houses within the third ring of Fuzhou to find out the correlation between green space accessibility and housing prices within the third ring of Fuzhou, and concluded that: (1) The residential houses within the third ring of Fuzhou are scattered in a circular pattern, whose density decreases from the first ring to the third ring; (2) The housing prices within the third ring of Fuzhou peaks at the city centre, higher than the outside ring, ranging between 18000 and $28000 / \mathrm{m}^{2}$. (3) The Gulou District has the highest grade of accessibility among houses within the third ring of Fuzhou while the Cangshan District was the poorest in this regard; (4) The residential houses within the third ring of Fuzhou are mainly graded as Very good and Good, with an overall high level of accessibility of parks; (5) There is a significant correlation between the walking time to the park and the housing price, the shorter the time consumption, the higher the housing price. Based on the above analysis, the paper proposed the following suggestions for the future urban planning and development of the real estate market, including: (1) Proper plan the entry and exit points of Gaogaishan Park, and add more entrances and exits to improve its accessibility; (2) Improve the urban road network and promote the construction of paths to shorten the distance between the green space and residence.

Based on ArcGIS shortest path, the paper proposed a practical approach to measure the impact of park accessibility on housing prices, providing references for the development of real estate market and the construction of an ecologically liveable city.

\section{References}

1. Gao Chunliang, Wei Houkai. Research on the Prediction of Urbanization Trend in China [J]. Contemporary Economic Sciences, 2013, 35 (04): $85-90+127$.

2. Gao Wenxiu, Fan Xiang, Zheng Fen, Yang Xiaochun. Spatial layout analysis of comprehensive park and its effective service area [J]. Urban Planning, 2017, 41 (11): 97-101 + 110 .

3. Guo Song, Fan Zeyuan, He Jiaqi, Li Zailiu. Park Accessibility of Xixiang Tang District in Nanning Based on Network Analysis Method [J]. China Garden, 2019, 35 (01): 68-72.

4. Zhou X, Parves Rana M. Social benefits of urban green space: A conceptual framework of valuation and accessibility measure-ments [J]. Management of Environmental Quality: An Interna-tional Journal, 2012, 23 (2): 173 -189.
5. Chen Qiuxiao, Hou Yan, Wu Shuang. Evaluation of the accessibility of green space in Shaoxing City Park from the perspective of fair opportunity [J]. 2016,36(3): 375-383. [Chen Qiuxiao, Hou Yan, Wu Shuang. Accessibility evaluation of urban parkgreen space in Shaoxing from the perspective of opportunity eq-uity. Scientia Geographica Sinica, 2016,36(3): 375-383.

6. Fan $\mathrm{P}, \mathrm{Xu} \mathrm{L}$, Yue $\mathrm{W}$ et al. Accessibility of public urban greenspace in an urban periphery: The case of Shanghai [J]. Land-scape and Urban Planning, 2017 (165): 177-192.

7. Sang Lijie, Shu Yonggang, Zhu Weiping, et al. Accessibility Analysis of Hangzhou Urban Recreational Green Space

8. Progress in Geographical Sciences, 2013,32 (6): 950-957. [Sang Lijie, ShuYonggang, Zhu Weiping et al. Analysis of accessibility of urbanleisure green space in Hangzhou. Progress in Geography, 2013, 32 (6) : 950-957.]

9. Yang J, Li C, Li Y et al. Urban green space, uneven develop-ment and accessibility: A case of Dalian's Xigang District [J]. Chinese Geographical Science, 2015, 25 (5): 644-656.

10. Sotoudehnia F, Comber L. Measuring perceived accessibility tourban green space: An integration of GIS and participatory map [J]. Agile, 2011, April 18-22.

11. Coombes E, Jones A P, Hillsdon M. The relationship of physicalactivity and overweight to objectively measured green space ac-cessibility and use [J]. Social Science \& Medicine, 2010, 70 (6): 816-822.

12. Dai D. Racial / ethnic and socioeconomic disparities in urbangreen space accessibility: Where to intervene? [J]. Landscape \& Urban Planning, 2011, 102 (4): 234-244.

13. Wendel HEW, Zarger RK, Mihelcic J R. Accessibility and us-ability: Green space preferences, perceptions, and barriers in arapidly urbanizing city in Latin America [J]. Landscape \& Ur-ban Planning, 2012, 107 (3): 272-282.

14. Christophercoutts, Markhorner, Timothychapin. Using geo-graphical information system to model the effects of greenspace accessibility on mortality in Florida [J]. Geocarto Interna-tional, 2010, 25 (6): 471-484.

15. Yin Haiwei, Kong Fanhua, Zong Yueguang. Evaluation of Urban Green Space Accessibility and Fairness [J]. Acta Ecologica Sinica, 2008,28 (7): 3375-3383. [Yin Haiwei, Kong Fanhua, Zong Yaoguang. Evaluation of accessibility and fairness of ur-ban green space. Acta Ecologica Sinica, 2008, 28 (7): 3375-3383.]

16. Yang Jun, Li Chuang, Li Yonghua, et al. Research on network reachability and service area green space spatial differentiation [J]. Bulletin of Surveying and Mapping, 2015 (3): 40-43. [Yang Jun, Li Chuang, Li Yonghua et al. Research on spatial differentiation of 
networkaccessibility and service scope. Bulletin of Surveying and Map-ping, 2015 (3): 40-43.]

17. Wei Ye, Xiu Chunliang, Gao Rui, et al. 2014. Green space accessibility evaluation in Shenyang City based on Gaussian two-step mobile search method [J]. Advances in Geographical Sciences, 2014, 33 (4), 479-487.

18. Wei Ye, Xiu Chunliang, Gao Rui et al. Evaluation of greenspace accessibility of Shenyang using Gaussian based 2-stepfloating catchment area method. Progress in Geography, 2014,33 (4), 479487.

19. Chen Ziyue. Study on the Spatialization of Demographic Data Based on the Classification of Residential Areas [J]. Geographical Spatial Information, 2016,14 (09): 47-48 + 52 + 7. [19] Zeng Zhan. Urban Population Spatial Distribution Research on Models and Empirical Methods [D]. Fuzhou University, 2014.

20. Xiao Jinghao. Evaluation of park green space service level and fairness based on big dataTaking Liuzhou City as an example [C]. China Urban Planning Institute, Dongguan Municipal People's Government. Rational Planning for Sustainable Development-2017 China Urban Planning Annual Meeting Proceedings (05 Urban Planning New Technology Application). China Urban Planning Society, Dongguan Municipal People's Government: China Urban Planning Society, 2017: 235-242.

21. Ma Lin, Lu Yuqi. Study on the urban green space landscape accessibility based on the road network structure: Taking the green space of Nanjing's main urban park as an example [J]. Chinese Garden, 2011, 27 (7): 92-96.

22. Zhang Guangliang. Research on Urban Park Green Space Accessibility Based on GIS Network Analysis [D]. Zhengzhou: Henan Agricultural University, 2012. 